\title{
Scale-wise Lyapunov exponent for bounded variation mappings
}

\author{
Zouhaier Dhifaoui ${ }^{1}$ \\ (c) Springer Nature Switzerland AG 2019
}

\begin{abstract}
The objective of this article is to study the properties of the maximal Lyapunov exponent (mLe) in the wavelet domain for one dimensional maps of bounded variation. In particular, using compactly supported wavelets, we expound the scale-dependence of the mLe for this class of maps. A simulation study involving two maps of bounded variation proves our theoretical results.
\end{abstract}

Keywords Function of bounded variation · Maximal Lyapunov exponent · Multiscale maximal Lyapunov exponent

\section{Introduction}

The Lyapunov exponent is the most useful diagnostic tool for chaos detection in dynamical systems. It is a quantitative measure of the sensitivity to initial condition phenomenon. Actually, a system with positive Lyapunov exponent is chaotic while a dissipative system is characterized by a negative Lyapunov exponent. This exponent is related to the called Kolmogorov entropy (capacity) [1], in fact, an estimation of the Kolmogorov entropy is given by the sum of all positive Lyapunov exponents [2]. The mLe can be used as an important tool in many applications such as detecting the presence of deterministic chaos in economic and financial databases (see for example $[3,4]$ ) or as predicting time series (see for example [5, 6]).

Over the past decades, there has been a big interest in wavelets as a technic allowing to separate the basic dynamics of a given signal. Also wavelets analysis offers a new time scale framework for the analysis, for the estimation and for the interpretation of various important statistical measures.

In this study, we present the definition of the scale-wise $\mathrm{mLe}$, and we explain that this measure tends to $-\infty$ as the wavelet level decomposition tends to $-\infty$ for one dimensional maps of bounded variation.
The rest of this research is organized as follows: a brief theoretical background on functions and maps of bounded variation is considered in Sect. 2. Basic principles of wavelet theory and wavelet-based characterization of bounded variation function are exposed in Sect. 3. In Sect. 4 we recall some definitions relating to the Lyapunov exponent and the Wolf estimator. Theoretical results are introduced in Sect. 5. Section 6 presents a simulation study validating the obtained results. Conclusions are outlined in Sect. 7.

\section{Function and mapping of bounded variation}

Before defining functions of bounded variation and presenting some of their properties, we must first evoke the total Jordan variation of a function $f$ [7] which is defined as follows:

Definition 2.1 (Camille Jordan variation) Suppose given a function $f:[a, b] \rightarrow \mathbb{R}$, and let $P=\left\{x_{0}, x_{1}, \ldots, x_{n}\right\}$ be any partition of the interval $[a, b]$, and let $V_{p}$ be such as:

$V(P)=\sum_{k=1}^{n}\left|f\left(x_{k}\right)-f\left(x_{k-1}\right)\right|$.

Zouhaier Dhifaoui, zouhaierdhifaoui@ymail.com | 'Department of Family and Community Medicine, Faculty of Medicine of Sousse, University of Sousse, Sousse, Tunisia. 
The total Jordan variation of $f$ on the interval $[a, b]$ is formally given by:

$V_{f}[a, b]=\sup _{P}\{V(P)\}$

where the supremum is taken over all possible partitions of $[a, b]$.

Definition 2.2 (Function of bounded variation) If $V_{f}[a, b]$ is finite we say that $f$ is of bounded variation on $[a, b]$ (in symbols we write $f \in B V[a, b])$.

If $f$ satisfies a Lipschitz condition on $[a, b]$, then $f$ is of bounded variation there. Also, any function of bounded variation on $[a, b]$ can be written as the difference of two increasing functions. Remark that this property reduces the theory of bounded variation functions to that of the bounded increasing functions. For more details on functions of bounded variation, we refer the reader to [8]. Let's review the definition of maps of bounded variation along with their basic properties. The following definition is due to Jordan [7].

Definition 2.3 (Bounded variation mapping) A mapping $f: \mathcal{E} \rightarrow \mathcal{X}$ is said to be of bounded variation if its total Jordan variation defined by Eq. (1) is finite, where $\mathcal{E}$ is a nonempty subset of $\mathbb{R}$ and $\mathcal{X}$ is the metric space.

Many theoretical results related to this type of mapping have been developed. In [9], the author has showed that, for a mapping of bounded variation defined on a subset of $\mathbb{R}$ with values in metric and normed spaces, the major aspects of the theory of real valued functions of bounded variation remains valid. Also, he demonstrated that $f: \mathcal{E} \rightarrow \mathcal{X}$ blue is a bounded variation mapping if and only if it is a composition of bounded nondecreasing function $\varphi$ and $\mathcal{X}$-valued mapping defined on the image of $\varphi$ satisfying the Lipschitz condition with Lipschitz constant lower to 1 . The reader can be referred to [9] for detailed proofs and discussions especially about the continuity properties of bounded variation mappings, for other results about the Holly principal selection which is verified for this type of mappings, and the relationship between the total variation of continuously differentiable mappings and the integral of the norm of their derivatives.

\section{Some facts on wavelet theory and wavelet-based characterization of bounded variation function}

A wavelet $\psi$ is a function belonging to $L^{2}(\mathbb{R})$ satisfying: $\int \psi(t) d t=0$

There are several families of wavelet functions that belong to the space $\mathcal{C}^{r}$ where $r \geq m$ and $m$ is the number of vanishing moments of $\psi$. We say that $\psi(t)$ has $m$ vanishing moments if it satisfies:

$\int t^{k} \psi(t) d t=0, \forall k=0, \ldots, m-1$.

In that case, $\psi$ is orthogonal to any polynomial of degree less than $m$. The most famous example of wavelets with vanishing moments is the Daubechies compactly supported wavelets [10].

A key concept in wavelet analysis is that an orthonormal basis of $L^{2}(\mathbb{R})$ can be generated from the wavelet function $\psi$ in the following manner:

$\psi_{j, k}(x)=2^{j / 2} \psi\left(2^{j} x-k\right),(j, k) \in \mathbb{Z}^{2}$,

allowing to decompose any finite energy function $f$ as follows:

$f(x)=\sum_{j=-\infty}^{+\infty} \sum_{k=-\infty}^{+\infty} d_{j, k} \psi_{j, k}(x)$

where $d_{j, k}=\int f(x) \psi_{j, k}(x) d x$ denotes the wavelets coefficient of the function $f$, whereas the partial sum

$f_{j}(x)=\sum_{k=-\infty}^{+\infty} d_{j, k} \psi_{j, k}(x)$

can be interpreted as the detail version $f$ at scale $2^{j}$.

Equation (2) can be rewritten by the following way:

$f(x)=\sum_{k=-\infty}^{+\infty} c_{j, k} \varphi_{j, k}(x)+\sum_{j=J_{0}}^{+\infty} \sum_{k=-\infty}^{+\infty} d_{j, k} \psi_{j, k}(x)$,

where $c_{j, k}$ are called scaling coefficients and $\varphi_{j, k}(x)=2^{j / 2} \varphi\left(2^{j} x-k\right)$. The scaling function $\varphi$ is such that:

$\varphi(x)=\sqrt{2} \sum_{k=-\infty}^{+\infty} g_{k} \varphi(2 x-k)$,

and is related to $\psi$ by:

$\psi(x)=\sqrt{2} \sum_{k=-\infty}^{+\infty} h_{k} \varphi(2 x-k)$.

Here $g$ and $h$ are respectively a low-pass filter and a highpass filter related by the relationship:

$h_{k}=(-1)^{1-k} g_{1-k}$. 
The reader can be referred to [11, 12] for more details about the wavelet analysis and the concept of multiresolution analysis. The latter being very important for wavelet bases derivation.

Now, let us focus on a wavelet characterization of bounded variation functions due to [11] and involving the absolute value of the wavelet coefficients $d_{j, k}$. Actually, a function $f$ is of bounded variation if and only if:

$$
\begin{gathered}
\sum_{k=-\infty}^{+\infty}\left|d_{j, k}\right| \leq A 2^{-j / 2}\|f\|_{V}, \text { where }\|f\|_{V} \\
=\int_{0}^{1}\left|f^{\prime}(x)\right| d x<+\infty \text { and } A>0 .
\end{gathered}
$$

\section{The Lyapunov exponent: Wolf estimator and scale-wise definition}

The Lyapunov exponent allows to study the chaoticity properties of a dynamical system by characterizing its sensitivity to its initial conditions. This sensitivity can be quantified as:

$\left|\Delta x_{t}\right| \approx\left|\Delta x_{0}\right| \exp (\lambda t)$

where $\Delta x_{t}$ is the distance separating two trajectories at time $t, \Delta x_{0}$ is the initial distance, and $\lambda$ is the mean rate of separation of the system's trajectories, $\lambda$ is called the Lyapunov exponent.

Let $(\mathcal{X}, f, \mu)$ be a dynamical system, where $\mathcal{X}$ is an Euclidean space with finite topological dimension. Suppose $f: \mathcal{X} \rightarrow \mathcal{X}$ is a transformation and $\mu$ is a probability measure on $\mathcal{X}$ (see chapter 3 of [13]). The Lyapunov exponent is formally defined as:

Definition 4.1 (The Lyapunov exponent) If the function $f$ which characterizes the dynamical system is differentiable, then the Lyapunov exponent for this system is given by the following quantity:

$\lambda=\lim _{n \rightarrow \infty} \frac{1}{n} \sum_{l=0}^{n}\left|f^{\prime}\left(x_{l}\right)\right|$

where $n$ indicates the number of points $x_{k}$ generated from the initial condition $x_{0}$ according to the equation of the system given by $x_{k+1}=f\left(x_{k}\right)$.

Many methods have been developed for estimating $\lambda$, such as those based on tangent space methods [14-17]. In this study, we take into consideration the best known and practical Wolf estimator [18] which computes the Lyapunov exponent from a scalar time series based on the use of Takens procedure. Suppose we have a time series $\left\{x_{i}\right\}_{i=1, \ldots, N}$. Then, based on this series of observations we construct
$N-(m-1) \tau$ delay state vectors by embedding $\left\{x_{i}\right\}_{i=1, \ldots, N}$ in a space of dimension $m$ as:

$\mathbf{x}_{i}^{m}=\left[x_{i}, x_{i+\tau}, x_{i+2 \tau}, \ldots, x_{i+(m-1) \tau}\right]^{t}, i=1, \ldots, N-(m-1) \tau$, where $\tau$ is an integer referred to a time lag and $m$ is usually referred to the embedding dimension. By this embedding the fractal character of attractor may be perceived when calculating the Lyapunov exponent, and on the other hand Takens [19] demonstrates that the properties of the function $\Psi^{m}: \mathbb{R}^{m} \rightarrow \mathbb{R}^{m}$, which represents the evolution of system as $\Psi^{m}\left(\mathbf{x}_{i}^{m}\right)=\mathbf{x}_{i+\tau^{\prime}}^{m}$ are identical to that of unknown function $f$ when $m \geq 2 d+1$ where $d$ is the dimension of deterministic system.

The algorithm of Wolf is an iterative procedure as follows: starting from an arbitrary point $\mathbf{x}_{1}^{m}$, we should then find the nearest neighbor $\mathbf{x}_{1}^{m^{\prime}}$ in the Euclidean sense to the initial point such as $\left\|\mathbf{x}_{1}^{m^{\prime}}-\mathbf{x}_{1}^{m}\right\|=e_{0}<e_{\min }$. After that, we draw the system's dynamics using initial points $\mathbf{x}_{1}^{m}$ and $\mathbf{x}_{1}^{m^{\prime}}$. If the distance $e_{0}^{\prime}$ between the two trajectories exceeds some value $e_{\text {max }}$, we stop and we calculate the ratio $e_{0}^{\prime} / e_{0}$. After a time of tracing $T_{0}, \mathbf{x}_{1}^{m}$ has evolved in $\mathbf{x}_{2}^{m}$ and $\mathbf{x}_{1}^{m^{\prime}}$ to $\mathbf{x}_{1}^{m^{\prime \prime}}$, then, we search for another starting point $\mathbf{x}_{1}^{m^{\prime \prime}}$ which is close to $\mathbf{x}_{1}^{m}$ and shifted in the direction of the vector $\mathbf{x}_{1}^{m^{\prime}}-\mathbf{x}_{1}^{m}$, and we denote by $e_{1}$ the distance separating $\mathbf{x}_{1}^{m}$ and $\mathbf{x}_{1}^{m^{\prime}}$. Iterating this process, we get a sequence of new points separated by distances $e^{\prime}{ }_{0}, e^{\prime}{ }_{1}, \ldots$. Now the $m L e$ is given by:

$\hat{\lambda}=\frac{\sum_{k=0}^{K} \log \left(\frac{e_{k}^{\prime}}{e_{k}}\right)}{\sum_{k=0}^{K} T_{k}}$,

where $K$ is the number of the algorithm's iterations. This procedure is illustrated by Fig. 1 where the distances $e^{\prime}$ are given by dashed line segment and noted by $L^{\prime}$, while the distances $e$ are noted by $L$ and given by continuous line segment.

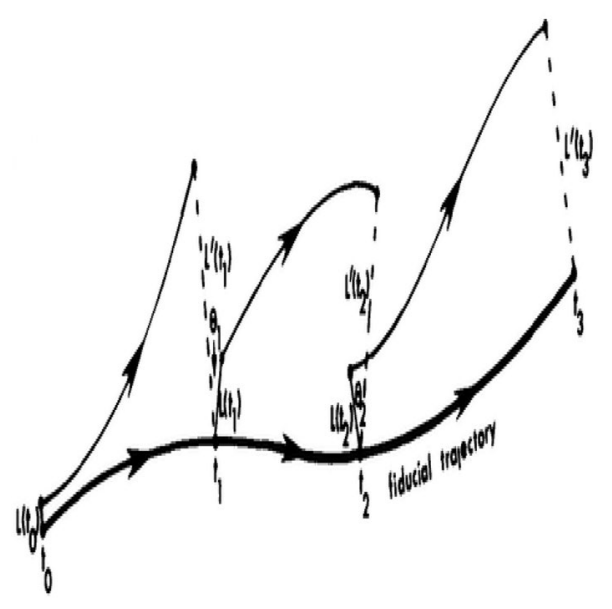

Fig. 1 Wolf procedure to estimate mLe ([18]) 
Definition 4.2 (Multiscale Lyapunov exponent) Let $f$ be a one-dimensional bounded variation map. The wavelet mLe associated to the scale $j$ is defined as follows:

$\lambda_{j}=\lim _{n \rightarrow \infty} \frac{1}{n} \sum_{l=1}^{n-1} \log \left(\left|f_{j}^{\prime}\left(x_{l}\right)\right|\right)$,

where $f_{j}^{\prime}$ denotes the first derivative of the wavelet details $f_{j}(x)$ given by Eq. (3) of the map $f$ at scale $j$.

\section{Behavior of the $m L e$ in the wavelet domain}

Proposition 5.1 Let $f$ be a one-dimensional differentiable map of bounded variation on $[0,1]$. Let $\psi$ be a compactly supported wavelet that belongs to $\mathcal{C}^{1}$. Then the scale $j$ wavelet mLe satisfies:

$\lim _{j \rightarrow-\infty} \lambda_{j}=-\infty$.

Proof For differentiable map, the scale $j$ wavelet maximal Lyapunov exponent can be written as:

$\lambda_{j}=\lim _{n \rightarrow \infty} \frac{1}{n} \log \left(\prod_{l=1}^{n-1}\left|f_{j}^{\prime}\left(x_{l}\right)\right|\right)$.

Now, using that $\psi$ is compactly supported and belongs to $\mathcal{C}^{1}$, then $\left|f_{j}^{\prime}\left(x_{l}\right)\right| \leq \sum_{k \in \mathbb{Z}}\left|d_{j, k}\right|\left|\psi_{j, k}^{\prime}\left(x_{l}\right)\right|$ and $\left|\psi_{j, k}^{\prime}\left(x_{l}\right)\right|=\left|\left(2^{j / 2} \psi\left(2^{j} x_{l}-k\right)\right)^{\prime}\right|=\left|2^{3 j / 2} \psi^{\prime}\left(2^{j} x_{l}-k\right)\right| \leq C 2^{3 j / 2}$ where $C$ is a real constant, then:

$\left|f_{j}^{\prime}\left(x_{l}\right)\right| \leq C 2^{3 j / 2} \sum_{k \in \mathbb{Z}}\left|d_{j, k}\right|$.

Using the relation (4), we have:

$\left|f_{j}^{\prime}\left(x_{l}\right)\right| \leq K 2^{j}$,

then, $\lambda_{j}=\lim _{n \rightarrow \infty} \frac{1}{n} \log \left(\prod_{l=1}^{n-1}\left|f_{j}^{\prime}\left(x_{l}\right)\right|\right) \leq \lim _{n \rightarrow \infty} \frac{1}{n} \log \left(\prod_{l=1}^{n-1} K 2^{j}\right)$.

Using that:

$$
\begin{aligned}
\log \left(\prod_{l=1}^{n-1} K 2^{j}\right) & =\sum_{l=1}^{n-1} \log (K)+j \sum_{l=1}^{n-1} \log (2) \\
& =(n-1)(\log (K)+j \log (2)) .
\end{aligned}
$$

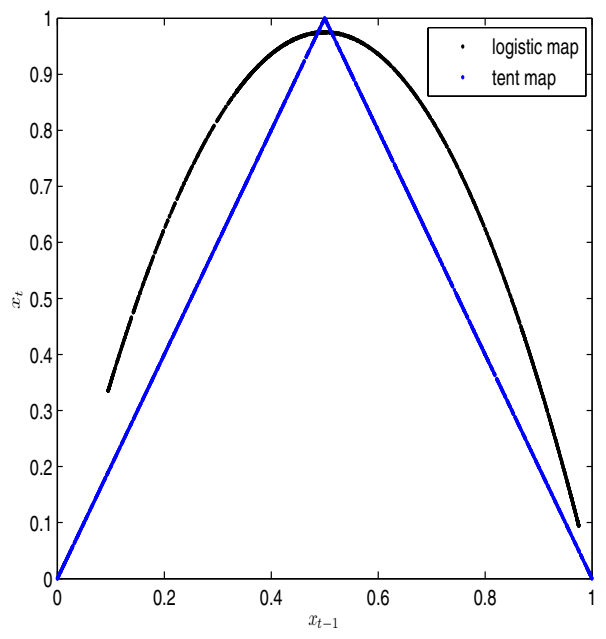

Fig. 2 The attractor of logistic and tent maps

Consequently, we have:

$\lambda_{j} \leq \lim _{n \rightarrow \infty} \frac{n-1}{n}(\log (K)+j \log (2)) \rightarrow-\infty$ when $j \rightarrow-\infty$.

Therefore, $\lambda_{j} \rightarrow-\infty$ when $j \rightarrow-\infty$.

\section{Numerical simulation}

To confirm the result of Sect. 5, we perform a simulation study by applying the Wolf estimator to two mappings of bounded variation on $[0,1]$, the first one is the logistic map defined by $f(x)=a x(1-x)$, the second one is the tent map defined by $f(x)=\mu x$ if $x<1 / 2$ and $\mu(1-x)$ if $x \geq 1 / 2$. We synthesize a signal of 2048 points from each

Table 1 Estimation of mLe for logistic and tent maps for associated level decomposition

\begin{tabular}{lll}
\hline Level $j$ & $\hat{\lambda}_{j}$ \\
\cline { 2 - 3 } & Logistic map & Tent map \\
\hline 11 & $1.4013 \mid 0.1403$ & $2.3071 \mid 0.1952$ \\
10 & $1.7635 \mid 0.2000$ & $2.1012 \mid 0.2127$ \\
9 & $1.4494 \mid 0.1562$ & $1.2852 \mid 0.1487$ \\
8 & $0.7493 \mid 0.0751$ & $0.4443 \mid 0.0551$ \\
7 & $0.5512 \mid 0.0634$ & $0.2835 \mid 0.0338$ \\
6 & $0.3208 \mid 0.0309$ & $0.2791 \mid 0.0316$ \\
5 & $0.0325 \mid 0.0325$ & $0.1834 \mid 0.0264$ \\
4 & $0.0761 \mid 0.0761$ & $0.1468 \mid 0.0169$ \\
3 & $0.0821 \mid 0.0821$ & $0.2593 \mid 0.0131$ \\
2 & $0.0083 \mid 0.0083$ & $0.0847 \mid 0.0057$ \\
1 & $0.0450 \mid 0.0450$ & $0.1107 \mid 0.0039$ \\
\hline
\end{tabular}


function by setting $a=3.9, \mu=4.1$ and $x_{0}=0.1$ as an initial condition. Figure 2 represents two attractors of signals generated using the chosen parameters for the logistic and the tent maps. We determine the details parts $f_{j}$ of the two signals for $j=1, \ldots, 11$ using a Daubechies wavelet with 4 vanishing moments. Then, we compute the $\mathrm{mLe}$ using the procedure cited in Sect. 4 by choosing $e_{\max }$ equal to $10 \%$ of the extent of time series and $e_{\min }=e_{\max } / 10$ [18], $\tau=1$ and embedding dimension $m=2$. Here the $\mathrm{mLe}$ for the $j$-level decomposition is computed for 1000 times using a Matlab code proposed in [20] where the starting initial condition is choosed randomly. The results of this study are summarized in Table 1, where for each resolution level $j$ the value on the left of the vertical bar is the mean value of 1000 replications and the value on the right is the associated standard deviation. All this numerical results are illustrated by Fig. 3a.

We notice from Table 1 a decline behavior of the mean value of the 1000 estimated values of the $\mathrm{mLe}$ at the eleven levels of decomposition whereas the values of

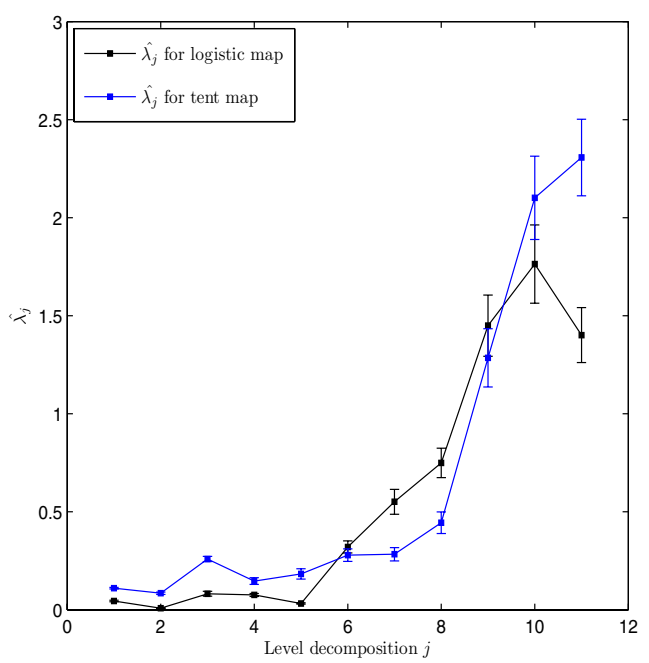

(a)

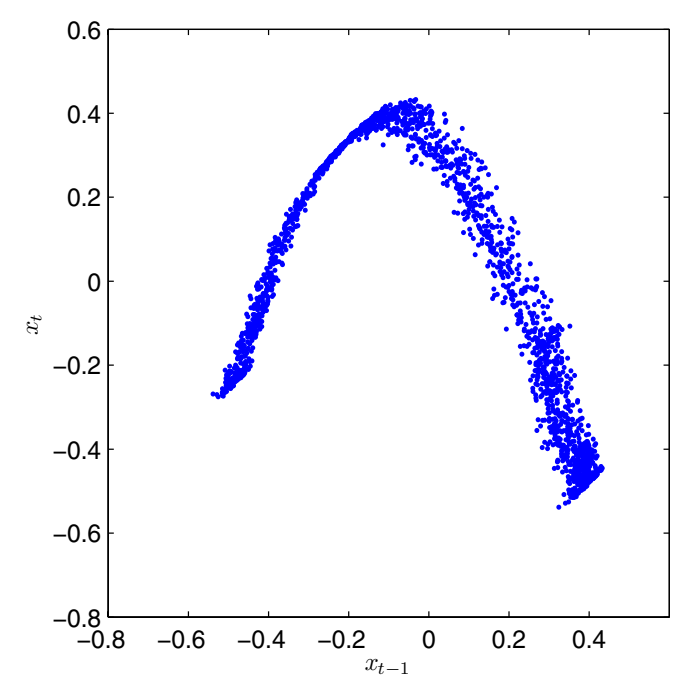

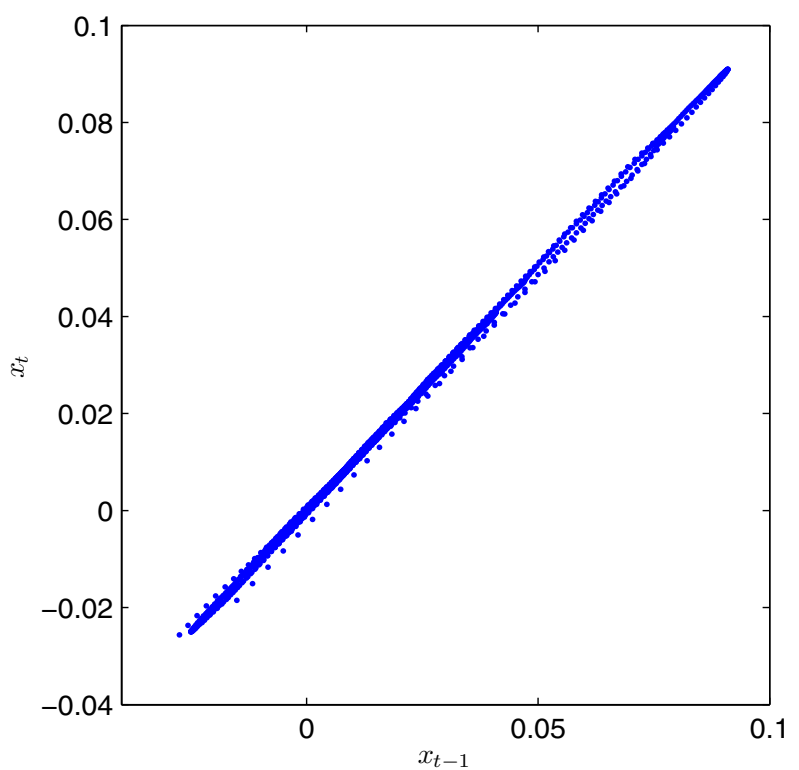

(c)

(b)

Fig. 3 a Evolution of $\hat{\lambda}_{j}$ versus level decomposition $j$ for logistic and tent maps, b reconstructed attractor of $f_{6}+f_{7}+\cdots f_{11}$ for logistic signal, $\mathbf{c}$ reconstructed attractor of $f_{1}+f_{2}+\cdots f_{5}$ for logistic signal 
the standard deviations are near zeros. But no negative value of the $\mathrm{mLe}$ for small level decomposition using that the Wolf estimator give only a positive value [18]. These results confirm that the small level decomposition corresponds to the noise part of the time series characterized by high frequency and no sensitivity to initial condition in the phase space, using that this level have a negative $\mathrm{mLe}$. For a very high level decomposition, the mLe is large positive, indicating the presence of sensitivity to initial condition phenomenon, with a view to serve notice of chaotic dynamic of this level which corresponds to the global behavior of the observations which is characterized by small frequency. For chaotic logistic process, these results are confirmed by reconstructed attractor for the sum of levels decomposition having a high value of $\mathrm{mLe}$ which correspond to levels $6,7,8,9,10$ and 11 given by Fig. $3 \mathrm{~b}$ when we see an attractor similar to that of studied signal. But for the sum of small levels decomposition 1,2, 3,4 and 5 having a zero $\mathrm{mLe}$, Fig. $3 \mathrm{c}$ shows no presence of an attractor like that's of logistic signal, then all small levels decomposition do not capture a chaotic behavior in the original studied chaotic signal.

\section{Conclusion}

In this work, we analyzed the characteristics of the multiscale $\mathrm{mLe}$ for a bounded variation maps. We have demonstrated that this exponent goes to $-\infty$ as the level decomposition $j$ tends to $-\infty$ for this type of mappings. These theoretical results are confirmed by a simulation study applying the well known Wolf estimator to the logistic and the tent maps.

\section{Compliance with ethical standards}

Conflict of interest The author declares there is no conflict of interest.
2. Pesin $Y$ (1977) Characteristic Lyapunov exponents and smooth ergodic theory. Russ Math Surv 32:55

3. Panas E, Ninni V (2000) Are oil markets chaotic? A non-linear dynamic analysis. Energy Econ 22:549-568

4. Bask M (1996) Dimensions and Lyapunov exponents from exchange rate series. Chaos Solitons Fractals 07:2199-2214

5. Ziehmann C, Smith L, Kurths J (2000) Localized Lyapunov exponents and the prediction of predictability. Phys Lett $A$ 271:237-251

6. Guégan D, Leroux J (2009) Forecasting chaotic systems: the role of local Lyapunov exponents. Chaos Solitons Fractals 41:2401-2404

7. Schwartz L (1967) Analyse mathématique: cours, vol 2. Hermann, Paris

8. Grady N (2009) Functions of bounded variation. Working paper

9. Chistyakov VV (1997) On mappings of bounded variation. J Dyn Control Syst 3:261

10. Daubechies I (1992) Ten lectures on wavelets. Society for Industrial and Applied Mathematics, Philadelphia

11. Mallat S (1989) A theory for multiresolution signal decomposition: the wavelet representation. IEEE Trans Pattern Anal Mach Intell 11:674-693

12. Mallat S (1999) A wavelet tour of signal processing. Elsevier Amsterdam

13. Robinson C (1999) Dynamical systems: stability, symbolic dynamics, and chaos. CRC Press, Boca Raton

14. Briggs K (1990) An improved method for estimating Liapunov exponents of chaotic time series. Phys Lett A 151:27-32

15. Eckmann JP, Ruelle D (1992) Fundamental limitations for estimating dimensions and Lyapunov exponents in dynamical systems. Phys D Nonlinear Phenom 56:185-187

16. Eckmann JP, Ruelle D (1985) Ergodic theory of chaos and strange attractors. Rev Mod Phys 57:617-656

17. Ellner SP, Ronald Gallant A, McCaffrey DF, Nychka DW (1991) Convergence rates and data requirements for Jacobian-based estimates of Lyapunov exponents from data. Phys Lett $A$ 153:357-363

18. Wolf A, Swift J, Swinney H, Vastano J (1985) Determining Lyapunov exponents from a time series. Phys D 16:285-317

19. Floris T (2006) Dynamical systems and turbulence. Springer, Berlin

20. Bovy J (2004) Lyapunov exponents and strange attractors in discrete and continuous dynamical systems. Theoretical physics project. School of Natural Sciences Einstein Drive, New Jersey

Publisher's Note Springer Nature remains neutral with regard to jurisdictional claims in published maps and institutional affiliations.

\section{References}

1. Pincus S (1991) Approximate entropy as a measure of system complexity. Proc Natl Acad Sci 88:2297-2301 